\title{
Invariant subspace problem and compact operators on non-Archimedean Banach spaces
}

\author{
M. BABAhmed, A. El ASRI \\ Department of Mathematics, University of Moulay Ismail \\ Faculty of Sciences, Meknes, Morocco \\ m.babahmed@umi.ac.ma, azzedine.elasri1@usmba.ac.ma
}

Received July 25, 2020

Presented by Manuel González

Accepted September 21, 2020

\begin{abstract}
In this paper, the invariant Subspace Problem is studied for the class of non-Archimedean compact operators on an infinite-dimensional Banach space $\mathbf{E}$ over a nontrivial complete nonArchimedean valued field $\mathbb{K}$. Our first main result (Theorem 9 asserts that if $\mathbb{K}$ is locally compact, then each compact operator on $\mathbf{E}$ possessing a quasi null vector admits a nontrivial hyperinvariant closed subspace. In the second one (Theorem 17), we prove that each bounded operator on $\mathbf{E}$ which contains a cyclic quasi null vector can be written as the sum of a triangular operator and a compact shift operator, each one of them possesses a nontrivial invariant closed subspace. Finally, we conclude that if $\mathbb{K}$ is algebraically closed, then every compact operator on $\mathbf{E}$ either has a nontrivial invariant closed subspace or is a sum of upper triangular operator and shift operator, each of them is compact and has a nontrivial invariant closed subspace.
\end{abstract}

Key words: Invariant subspace, hyperinvariant subspace, compact operator, $t$-orthogonal basis, quasi null vector, triangular operator, shift operator.

AMS Subject Class. (2010): 47A15, 47S10, 46S10.

\section{INTRODUCTION}

One of the most important problem in operator theory is the invariant subspace problem, which is concerned with the existence of invariant subspaces of a bounded operator on separable infinite-dimensional Banach space. This problem is one of the best-known unresolved problem in functional analysis. It is not clear exactly when the problem was formally posed. Some believe that the interest aroused by this problem stems from a Beurling's paper [6] and an unpublished work by von Neumann in which he had shown that compact operators on a complex Hilbert space of dimension at least 2 have nontrivial invariant closed subspaces. Since then, research into the existence of invariant subspaces is intensively launched. A number of authors worked on extending this result and significant progress was made from then until now. 
In 1954, Aronszajn and Smith [3] generalized the result obtained by von Neumann to compact operators on an infinite-dimensional Banach space over the complex field $\mathbb{C}$.

In 1966, Bernstein and Robinson [5], using nonstandard analysis, proved that each polynomially compact operator on a complex Hilbert space has a nontrivial invariant closed subspace. In the same year, Halmos [10] gave a proof of the same result by a similar method, but avoiding the nonstandard analysis tools.

In 1968, Arveson and Feldman [4] proved that if $T$ is a quasitriangular operator on a Hilbert space (an operator satisfying $\lim _{n}\left\|T P_{n}-P_{n} T P_{n}\right\|=0$ for some sequence $\left(P_{n}\right)_{n}$ of orthogonal projection operators which converges strongly to the identity $I$ ), then $T$ has a nontrivial invariant closed subspace if the closed algebra generated by $T$ and $I$ contains a nonzero compact operator.

In 1973, Pearcy and Salinas [14] proved that if $T$ is a quasitriangular operator on a Hilbert space $H$ and $\mathbf{R}(T)$, the norm closure of the rational functions of $T$, contains a nonzero compact operator, then there exists a nontrivial invariant closed subspace under all operators in $\mathbf{R}(T)$. In the same year, appeared the famous theorem of Lomonosov [12]. This theorem generalized all the preceding results. Lomonosov showed that each operator on a Banach space that commutes with a nonzero compact operator has a nontrivial hyperinvariant closed subspace. He used new techniques in his proof like Schauder's fixed point theorem. Then, the theorems due to von Neumann, Aronszajn and Smith, Bernstein and Robinson, become direct corollaries of this theorem. The Lomonosov's theorem obtained was a more general result than anyone had even hoped to be able to prove. In the same year, Hilden found a proof of Lomonosov's theorem without using Schauder's fixed point theorem, and with only the most elementary notions of functional analysis (see [16, p. 158]).

In 1977, Michaels [13 presented the Hilden's proof with more simplifications, making it accessible to nonspecialists. At this time, the Lomonosov result seemed so strong that some authors wondered if his hypothesis (commutativity with a compact operator) could even be true for all operators. Unfortunately, in 1980 Hadwin, Nordgren, Radjavi and Rosenthal [9] constructed an operator not satisfying such hypothesis. In the non-Archimedean setting, in 2008 Sliwa [20] proved that every infinite-dimensional non-Archimedean Banach space of countable type admits a bounded operator without a nontrivial invariant closed subspace. 
In this paper, taking inspiration from Hilden's proof techniques of Lomonosov's Theorem, we prove that each compact operator on an infinitedimensional Banach space over a locally compact non-Archimedean valued field in which there exists a quasi null vector have a nontrivial hyperinvariant closed subspace (Theorem 9). We prove also that each bounded operator on infinite-dimensional non-Archimedean Banach space which contains a cyclic quasi null vector can be written as the sum of a triangular operator and a compact shift operator, each one of them has a nontrivial invariant closed subspace (Theorem 17).

\section{Preliminary and auxiliary Results}

In this section, we collect some auxiliary results of non-Archimedean analysis that we need later.

Let $\mathbb{K}$ be a field, a valuation on $\mathbb{K}$ is a function $||:. \mathbb{K} \rightarrow[0, \infty)$ such that for all $a, b \in \mathbb{K}$ :

(i) $|a|=0$ if and only if $a=0$;

(ii) $|a b|=|a||b|$;

(iii) $|a+b| \leq|a|+|b|$ (the triangle inequality).

The pair $(\mathbb{K},||$.$) is called a valued field. We frequently write \mathbb{K}$ instead of $(\mathbb{K},||$.$) . A valuation |$.$| on \mathbb{K}$ is:

- non-trivial if $|a| \neq 1$ for some $a \in \mathbb{K} \backslash\{0\}$;

- non-Archimedean if $|a+b| \leq \max \{|a|,|b|\}$ for all $a, b \in \mathbb{K}$;

- complete if $\mathbb{K}$ is complete with respect to the metric $d(a, b)=|a-b|$ induced by the valuation |.|.

For fundamentals on non-Archimedean valued field we refer to [15, 21].

Throughout this paper, $\mathbb{K}:=(\mathbb{K},||$.$) is a non-Archimedean non-trivially$ valued complete field. The closed unit ball and the open unit ball in $\mathbb{K}$ are respectively $\overline{\mathbf{B}}_{\mathbb{K}}=\{\lambda \in \mathbb{K}:|\lambda| \leq 1\}$ and $\mathbf{B}_{\mathbb{K}}=\{\lambda \in \mathbb{K}:|\lambda|<1\}$. The residue class field of $\mathbb{K}$ is $\mathbb{k}=\overline{\mathbf{B}}_{\mathbb{K}} / \mathbf{B}_{\mathbb{K}}$. We say that $\mathbb{K}$ is locally compact if each point has a compact neighbourhood. So, clearly $\mathbb{K}$ is locally compact if, and only if, the unit ball $\overline{\mathbf{B}}_{\mathbb{K}}$ of $\mathbb{K}$ is compact. And we have the following characterization of a locally compact field:

ThEOREM 1. ([15, P. 9]) $\mathbb{K}$ is locally compact if, and only if, the valuation is discrete and the residue class field is finite. 
The most known example of a non-Archimedean valued field is the field of $p$-adic numbers $\mathbb{Q}_{p}$ which is locally compact (see [15, p. 9]).

We say that $\mathbb{K}$ is spherically complete if any decreasing sequence of closed balls in $\mathbb{K}$ has a non-empty intersection (see [15, p. 4]).

Now let $\mathbf{E}$ be a vector space over $\mathbb{K}$. A norm $\|$.$\| on \mathbf{E}$ is called nonArchimedean if it satisfies the following strong inequality:

$$
\|x+y\| \leq \max \{\|x\|,\|y\|\} \quad \text { for all } x, y \in \mathbf{E} .
$$

It is easy to verify that if $\|$.$\| is a non-Archimedean norm in \mathbf{E}$, then for $x, y \in \mathbf{E}$ with $\|x\| \neq\|y\|$ we have $\|x+y\|=\max \{\|x\|,\|y\|\}$. We say that $\mathbf{E}:=(\mathbf{E},\|\|$. is a non-Archimedean Banach space if the norm $\|$.$\| is non-Archimedean on \mathbf{E}$, and it is complete with the topology induced by the metric $d(x, y)=\|x-y\|$.

If $\mathbf{E}$ contains a countable set $Y$ such that its linear hull is dense in $\mathbf{E}$, i.e., $\overline{[Y]}=\mathbf{E}$, then we say that $\mathbf{E}$ is of countable type. Let $t \in(0,1], \mathbf{A}$ vector $x \in \mathbf{E}$ is $t$-orthogonal (orthogonal, when $t=1$ ) to $y \in \mathbf{E} \backslash\{\mathbf{0}\}$ if $d(x, \mathbb{K} y):=\operatorname{dist}(x, \mathbb{K} y) \geq t\|x\|$, where $\operatorname{dist}(x, D):=\inf _{d \in D}\|x-d\|(D \subseteq \mathbf{E})$ and $\mathbb{K} y$ denotes a one-dimensional linear subspace generated by the element $y$. We note $x \perp_{t} y$. By the principle of van Rooij (see [15, p. 23]), if $x \perp_{t} y$ then

$$
\|\lambda x+\mu y\| \geq t \max \{\|\lambda x\|,\|\mu y\|\} \quad \text { for all } \lambda, \mu \in \mathbb{K} .
$$

So, the $t$-orthogonality is a symmetric relation. We say that a subset $X$ of $\mathbf{E} \backslash\{0\}$ is $t$-orthogonal (orthogonal, when $t=1$ ) system if for each $x \in X$, $x \perp_{t} y$ for all $y \in[X \backslash\{x\}]$. Clearly, a subset $X=\left\{x_{1}, x_{2}, \ldots, x_{n}, \ldots\right\} \subseteq \mathbf{E} \backslash\{0\}$ is $t$-orthogonal if, and only if, each finite subset of $X$ is $t$-orthogonal, i.e.,

$$
\left\|\sum_{i=1}^{m} \lambda_{i} x_{i}\right\| \geq t \max _{i=1}^{m}\left\|\lambda_{i} x_{i}\right\|
$$

for all $\lambda_{1}, \ldots, \lambda_{m} \in \mathbb{K}$ and all $x_{1}, \ldots, x_{m} \in X$, where $x_{i} \neq x_{j}$ for $i \neq j$. And $\left\{x_{1}, x_{2}, \ldots, x_{n}, \ldots\right\}$ is orthogonal if, and only if,

$$
\left\|\sum_{i=1}^{m} \lambda_{i} x_{i}\right\|=\max _{i=1}^{m}\left\|\lambda_{i} x_{i}\right\| .
$$

If $A$ and $B$ are two subset of $\mathbf{E}$, we say that $A$ and $B$ are $t$-orthogonal if for every $(a, b) \in A \times B$ we have $a \perp_{t} b$. Then, we write $A \perp_{t} B$. A sequence $\left(x_{n}\right)_{n}$ in $\mathbf{E}$ is called a t-orthogonal (orthogonal, when $t=1$ ) basis of $\mathbf{E}$ if $\left\{x_{1}, x_{2}, \ldots, x_{n}, \ldots\right\}$ is $t$-orthogonal and every $x \in \mathbf{E}$ has an expansion 
$x=\sum_{n=1}^{+\infty} \lambda_{n} x_{n}$, where $\lambda_{n} \in \mathbb{K}$ for every $n \in \mathbb{N}$. The basis is orthonormal if, in addition, $\left\|x_{n}\right\|=1$ for all $n \in \mathbb{N}$.

For more background on non-Archimedean normed space over valued field and more details we refer the reader to [21, 18. Now, we recall the following important theorem:

Theorem 2. ([15, P. 30]) If $\mathbf{E}$ is of countable type, then for each $t \in$ ]0, 1 , $\mathbf{E}$ has a t-orthogonal basis. In addition, if $\mathbb{K}$ is spherically complete, $\mathbf{E}$ has an orthogonal basis.

We call a nonempty subset $X$ of $\mathbf{E}$ absolutely convex if $a x+b y \in X$ for all $x, y \in X$ and $a, b \in \bar{B}_{\mathbb{K}}$. Let $X$ be any subset of $\mathbf{E}$, $\operatorname{By~} \operatorname{Co}(X)$ we denote the absolutely convex hull of $X$, which is the smallest absolutely convex set in $\mathbf{E}$ that contains $X$. A subset $X$ of $\mathbf{E}$ is said to be compactoid if for every $\epsilon>0$ there exists a finite set $A \subset \mathbf{E}$ such that $X \subset B(0, \epsilon)+\operatorname{Co}(A)$ (where $B(0, \epsilon)=\{x \in \mathbf{E}:\|x\| \leq \epsilon\})$. Amice [2] proved that if $\mathbb{K}$ is locally compact, then a subset of $\mathbf{E}$ is compactoid if, and only if, it is precompact, then each complete compactoid subset of $\mathbf{E}$ is compact. It's easy to see that if $\mathbb{K}$ is not locally compact, any convex set (a translation of absolutely convex set) in $\mathbf{E}$ containing at least two points, is not compact.

An operator on $\mathbf{E}$ is a linear map $T: \mathbf{E} \rightarrow \mathbf{E}$. By $\mathbf{B}(\mathbf{E})$ we denote the algebra of all bounded operators on $\mathbf{E}$.

Now, we recall the definitions of completely continuous and compact operators on non-Archimedean Banach space.

Definition 3. Let $T$ be an operator on $\mathbf{E}$, we say that $T$ is:

1. completely continuous if $T(B(0,1))$ has a compact closure [19, 7];

2. compact if $T(B(0,1))$ is a compactoid (see [21, p. 142]).

We note that the first definition have no sense if $\mathbb{K}$ is not locally compact, and the two definitions coincide when $\mathbb{K}$ is locally compact (see [21, p. 142]).

Similarly as in classical Hilbert space analysis, we have the following result:

Theorem 4. ([21, P. 142]) Let $T \in \mathbf{B}(\mathbf{E})$. Then $T$ is compact if, and only if, for every $\epsilon>0$, there exists an $S \in \mathbf{B}(\mathbf{E})$ such that $S$ is a finite rank operator and $\|T-S\| \leq \epsilon$.

This means that the compact operators are exactly the (norm) limits of finite rank operators. The spectral theory on compact operators in classical 
analysis can be successfully extended to the set of compact operators in nonArchimedean analysis, see [8, 17.

The spectrum $\sigma(T):=\{\lambda \in \mathbb{K}: T-\lambda I$ is not invertible $\}$ of a compact operator $T$ is at most countable with 0 as its only possible accumulation point, so in particular it is compact (see [17, p. 17]). Moreover, each nonzero element of the spectrum is an eigenvalue (see [17, p. 16]). One also has the Fredholm alternative (see [17, p. 15]). And also, as in classical analysis over complex field, if $\mathbb{K}$ is algebraically closed then every compact operator $T$ is spectral in the following sense: $\max \{|\lambda|: \lambda \in \sigma(T)\}=\lim _{n}\left\|T^{n}\right\|^{\frac{1}{n}}$. For more on compact operators, see [7, 8, 17, 19].

\section{Definitions AND MAin RESUlts}

We are now ready to state and prove the main results of this paper.

Definition 5. Let $T$ be an operator on a Banach space $\mathbf{E}$, and $\mathbf{M}$ a nontrivial $(\{0\} \varsubsetneqq \mathbf{M} \varsubsetneqq \mathbf{E})$ closed subspace of $\mathbf{E}$. We say that $\mathbf{M}$ is:

1. invariant if $T \mathbf{M} \subseteq \mathbf{M}$.

2. hyperinvariant if $\mathbf{M}$ is invariant for each element of $\{T\}^{\prime}=\{S \in \mathbf{B}(\mathbf{E})$ : $T S=S T\}($ the commutant of $T)$.

We note that a bounded operator on a Banach space is said locally quasinilpotent at a vector $x$ if $\lim _{n}\left\|T^{n} x\right\|^{\frac{1}{n}}=0$ [1]. We point out that $T$ can be not locally quasinilpotent on certain nonzero vector $x$, and $\liminf _{n}\left\|T^{n} x\right\|^{\frac{1}{n}}=0$. In this case we shall say that $x$ is $T$-quasi null vector. It turns out that this property can be a very useful tool for the study of invariant subspaces. Note that even a compact operator may not have a T-quasi null vector. For example, we consider the operator $T$ on $c_{0}\left(\mathbb{Q}_{p}\right)$ (the non-Archimedean Banach space of null sequences on $Q_{p}$ ) defined by:

$$
T e_{n}=p^{n} e_{n} \quad \text { for all } n \geq 1,
$$

where $\left(e_{n}\right)_{n}$ is the standard orthogonal basis of $c_{0}\left(\mathbb{Q}_{p}\right)$. It is clear that $T$ is compact. Let $x=\left(x_{k}\right)_{k}$ be a nonzero element of $c_{0}\left(\mathbb{Q}_{p}\right)$. Then, $x=\sum_{k \geq 1} x_{k} e_{k}$ and for each $n \geq 1, T^{n} x=\sum_{k \geq 1} x_{k} p^{n k} e_{k}$. Let $r \geq 1$ be such that $x_{r} \neq 0$. So

$$
\left\|T^{n} x\right\|=\max _{k \geq 1}\left|x_{k}\right|\left|p^{n k}\right| \quad \text { and } \quad\left\|T^{n} x\right\|^{\frac{1}{n}} \geq\left|x_{r}\right|^{\frac{1}{n}} \frac{1}{p^{r}} .
$$

Therefore, $T$ cannot have a $T$-quasi null vector. 
Lemma 6. Let $\left(\alpha_{n}\right)_{n}$ be a real sequence such that $\liminf _{n} \alpha_{n}^{\frac{1}{n}}=0$. Then, for each real $\beta$ we have $\liminf { }_{n} \beta^{n} \alpha_{n}=0$.

Proof. Let $\beta \in \mathbb{R}$. Then for each $n \geq 1$ we have $\left(\beta^{n} \alpha_{n}\right)^{\frac{1}{n}}=\beta \alpha_{n}^{\frac{1}{n}}$.

Proposition 7. $x$ is T-quasi null vector if, and only if, $x$ is $\lambda T$-quasi null vector for each $\lambda \in \mathbb{K} \backslash\{0\}$.

Proof. Let $\lambda \in \mathbb{K} \backslash\{0\}$. For each $n \geq 1,\left\|(\lambda T)^{n} x\right\|^{\frac{1}{n}}=|\lambda|\left\|T^{n} x\right\|^{\frac{1}{n}}$.

Lemma 8. ([15, P. 18]) Let $\mathbf{E}:=(\mathbf{E},\|\|$.$) be a normed space over a dis-$ cretely valued field $\mathbb{K}$. Then there is an equivalent norm $\|\cdot\|^{\prime}$ on $\mathbf{E}$ for which $\|.\|^{\prime}$ is solid (i.e., $\|\mathbf{E}\|^{\prime} \subseteq|\mathbb{K}|$ ).

We note that if $T$ is an operator on $(\mathbf{E},\|\|$.$) and \|.\|^{\prime}$ is another nonArchimedean norm on $\mathbf{E}$ that is equivalent to $\|$.$\| . Then, every T$-quasi null vector of $(\mathbf{E},\|\cdot\|)$ is also a $T$-quasi null vector of $\left(\mathbf{E},\|\cdot\|^{\prime}\right)$. Every closed invariant (resp. hyperinvariant) subspace of $T$ in $(\mathbf{E},\|\|$.$) is closed invariant (resp.$ hyperinvariant) subspace of $T$ in $\left(\mathbf{E},\|.\|^{\prime}\right)$. So with this argument and Lemma 8. we can, without loss of generality, assume that the non-Archimedean norm in $\mathbf{E}$ is solid.

Now we are ready to show that on non-Archimedean Banach space over a locally compact field every compact operator with a $T$-quasi null vector has a nontrivial hyperinvariant closed subspace.

Theorem 9. Let $(\mathbf{E},\|\|)$ be a non-Archimedean Banach space over a locally compact field $\mathbb{K}$ and $T$ be a compact operator on $\mathbf{E}$. If there exists a $T$-quasi null vector in $\mathbf{E}$, then there exists a nontrivial hyperinvariant closed subspace of $T$.

Proof. Without loss of generality, we can assume that $T$ is an injective operator, and $\|T\|=1$. Let $z$ be a $T$-quasi null vector in $\mathbf{E}$. So $\lim \inf _{n}\left\|T^{n} z\right\|^{\frac{1}{n}}=$ 0 . Let $\lambda_{0} \in \mathbb{K}$ be such that $\left\|T\left(\lambda_{0} z\right)\right\|>1$. And let $z_{0}=\lambda_{0} z$, so $\left\|z_{0}\right\|=$ $\|T\|\left\|z_{0}\right\| \geq\left\|T z_{0}\right\|>1$. For each $\lambda \in \mathbb{K}$, by Lemma 6 .

$$
\liminf _{n}|\lambda|^{n}\left\|T^{n} z_{0}\right\|=\left|\lambda_{0}\right| \liminf _{n}|\lambda|^{n}\left\|T^{n} z\right\|=0 .
$$

For each $x \in \mathbf{E}$, set

$$
\mathbf{H}_{x}:=\left\{S x: S \in\{T\}^{\prime}\right\} .
$$


Then, $\mathbf{H}_{x}$ is an hyperinvariant subspace of $T$. Then, so is $\overline{\mathbf{H}}_{x}$ for each $x$. Since for each $x \in \mathbf{E} \backslash\{0\}, \mathbf{H}_{x} \neq\{0\}$, we have the result unless $\overline{\mathbf{H}}_{x}=\mathbf{E}$ for each $x \in \mathbf{E} \backslash\{0\}$. So, assume a contradiction and suppose that we have this. Let $\mathbf{B}:=\overline{\mathbf{B}}\left(z_{0}, 1\right)$ be a closed unit ball in $\mathbf{E}$. For each $x \in \mathbf{E} \backslash\{0\}, z_{0} \in \mathbf{E}=\overline{\mathbf{H}}_{x}$, then there exist $y \in \mathbf{H}_{x}$ such that $\left\|y-z_{0}\right\| \leq 1$. And there exists $S \in\{T\}^{\prime}$ such that $y=S x$. Hence, $\left\|S x-z_{0}\right\| \leq 1$, and $S x \in \mathbf{B}$. Therefore,

$$
\mathbf{E} \backslash\{0\} \subseteq \bigsqcup_{S \in\{T\}^{\prime}}\{x \in \mathbf{E}: S x \in \mathbf{B}\} .
$$

For each $S \in\{T\}^{\prime}$, let $\mathbf{U}(\mathbf{S}, \mathbf{B}):=\{x \in \mathbf{E}: S x \in \mathbf{B}\}$. Clearly $\mathbf{U}(\mathbf{S}, \mathbf{B})$ is open in $E$ for each $S \in\{T\}^{\prime}$. Since $0 \notin B$, we have $0 \notin T(\mathbf{B})$. Then,

$$
T(\mathbf{B}) \subseteq \bigsqcup_{S \in\{T\}^{\prime}} \mathbf{U}(\mathbf{S}, \mathbf{B}) .
$$

Since $T$ is compact, there exist $S_{1}, \ldots, S_{n} \in\{T\}^{\prime}$ such that

$$
T(\mathbf{B}) \subseteq \bigsqcup_{i=1}^{n} \mathbf{U}\left(\mathbf{S}_{\mathbf{i}}, \mathbf{B}\right) .
$$

$T z_{0} \in T(\mathbf{B}) \Rightarrow$ there is $i_{1} \in\{1, \ldots, n\}$ such that $T z_{0} \in \mathbf{U}\left(\mathbf{S}_{\mathbf{i}_{1}}, \mathbf{B}\right) \Rightarrow S_{i_{1}} T z_{0} \in$ $\mathbf{B} \Rightarrow T S_{i_{1}} T z_{0} \in T(\mathbf{B}) \Rightarrow$ there is $i_{2} \in\{1, \ldots, n\}$ such that $T S_{i_{1}} T z_{0} \in$ $\mathbf{U}\left(\mathbf{S}_{\mathbf{i}_{2}}, \mathbf{B}\right) \Rightarrow S_{i_{2}} T S_{i_{1}} T z_{0} \in \mathbf{B}$. And we continue like this so much we would like. Then for each $k \geq 1$, there exist $S_{i_{1}}, \ldots, S_{i_{k}} \in\{T\}^{\prime}$ such that

$$
S_{i_{k}} T S_{i_{k-1}} T \cdots S_{i_{1}} T z_{0} \in \mathbf{B} .
$$

Since the non-Archimedean norm of $\mathbf{E}$ is solid, let $\lambda \in \mathbb{K} \backslash\{0\}$ be such that $|\lambda|=\max _{1 \leq j \leq k}\left\|S_{i_{j}}\right\|$. So, we have $\left(\lambda^{-1} S_{i_{k}}\right) \cdots\left(\lambda^{-1} S_{i_{1}}\right)(\lambda T)^{k}\left(z_{0}\right) \in \mathbf{B} \Rightarrow$ $\left\|\left(\lambda^{-1} S_{i_{k}}\right) \cdots\left(\lambda^{-1} S_{i_{1}}\right)(\lambda T)^{k}\left(z_{0}\right)-z_{0}\right\| \leq 1$. Since $\left\|z_{0}\right\|>1$, we have

$$
\left\|\left(\lambda^{-1} S_{i_{k}}\right) \cdots\left(\lambda^{-1} S_{i_{1}}\right)(\lambda T)^{k}\left(z_{0}\right)\right\|=\left\|z_{0}\right\|>1 .
$$

Since $\left\|\lambda^{-1} S_{i_{r}}\right\| \leq 1$ for all $1 \leq r \leq k$, we get $\left\|(\lambda T)^{k} z_{0}\right\|>1$. Hence, $|\lambda|^{k}\left\|T^{k} z_{0}\right\|>1$ for each $k \geq 1$, which is a contraction. And the result follows.

Remark 10. It is known that the closure of the range of a compact operator is of countable type (see [21, p. 134]). So, if the Banach space $\mathbf{E}$ is not of countable type, this closure is a nontrivial hyperinvariant closed subspace. 
We note that if $\mathbf{E}$ is not of countable type, then every operator $T$ on $\mathbf{E}$ does not have a cyclic vector (a vector $x \in \mathbf{E}$ such that $\overline{\left[T^{n} x, n \geq 0\right]}=\mathbf{E}$ ). And so for each nonzero $x$ in $\mathbf{E}, \overline{\left[T^{n} x, n \geq 0\right]}$ is a nontrivial closed invariant subspace of $T$. Therefore, without loss of generality, we assume throughout to the end of this paper that $\mathbf{E}$ is a non-Archimedean Banach space of countable type. The following lemma and theorem will be needed to prove Lemma 14 .

Lemma 11. ([21, P. 66]) Let $\mathbf{F}$ be a closed subspace of $\mathbf{E}$ and $a \in \mathbf{E} \backslash \mathbf{F}$. then for each $t \in] 0,1[$ there exist $e \in \mathbf{E}$ such that $[a]+\mathbf{F}=[e]+\mathbf{F}$ and $e$ is $t$-orthogonal to $\mathbf{F}$. Furthermore, if $\mathbb{K}$ is spherically complete, we can choose $e$ to be orthogonal to $\mathbf{F}$.

Theorem 12. ([15, P. 30]) Let $t \in] 0,1]$ and let $\left\{x_{n}, n \in \mathbb{N}\right\}$ be a $t$ orthogonal subset of $\mathbf{E}$. If $\overline{\left[x_{n}, n \in \mathbb{N}\right]}=\mathbf{E}$, then $\left\{x_{1}, x_{2}, \ldots\right\}$ is a t-orthogonal (orthogonal, when $t=1$ ) basis of $\mathbf{E}$.

Lemma 13. ([15, P. 28]) Let $e_{1}, \ldots, e_{n}$ be distinct nonzero vectors in a normed space $\mathbf{E}$. Let $\left.\left.t_{2}, t_{3}, \ldots, t_{n} \in\right] 0,1\right]$ be such that

$$
\left\{\begin{array}{l}
\operatorname{dist}\left(e_{2},\left[e_{1}\right]\right) \geq t_{2}\left\|e_{2}\right\| \\
\operatorname{dist}\left(e_{3},\left[e_{1}, e_{2}\right]\right) \geq t_{3}\left\|e_{3}\right\| \\
\quad \vdots \\
\operatorname{dist}\left(e_{n},\left[e_{1}, \ldots, e_{n-1}\right]\right) \geq t_{n}\left\|e_{n}\right\| .
\end{array}\right.
$$

Then $\left\{e_{1}, \ldots, e_{n}\right\}$ is $\left(t_{2} t_{3} \cdots t_{n}\right)$-orthogonal.

Lemma 14. Let $T$ be a bounded operator on $\mathbf{E}$. Suppose that there exists a $T$-quasi null vector in $\mathbf{E}$ which is cyclic. Then, for each $t \in] 0,1[$, there exists $\left(e_{n}\right)_{n}$, a $t$-orthogonal basis of $\mathbf{E}$ such that:

1. $T\left[e_{1}, \ldots, e_{n}\right] \subseteq\left[e_{1}, \ldots, e_{n+1}\right]$ for all $n \geq 1$;

2. $\liminf \operatorname{in}_{n} d\left(T\left(e_{n}\right),\left[e_{1}, \ldots, e_{n}\right]\right)=0$.

Proof. Let $z$ be a cyclic $T$-quasi null vector in $\mathbf{E}$. So, we have

$$
\begin{aligned}
& \mathbf{E}=\overline{\left[z, T z, T^{2} z, \ldots\right]}, \\
& \liminf _{n}\left\|T^{n} z\right\|^{\frac{1}{n}}=0 .
\end{aligned}
$$


Let $t \in] 0,1\left[\right.$. For each $n \geq 1$, let $F_{n}:=\left[z, T z, T^{2} z, \ldots, T^{n-1} z\right]$. Choose $\left.t_{2}, t_{3}, \cdots \in\right] 0,1\left[\right.$ such that $t \leq \prod_{i>2} t_{i}$.

Let $e_{1}=z, T z \in \mathbf{E} \backslash \mathbf{F}_{\mathbf{1}}$, then by Lemma 11, there exists $e_{2} \in \mathbf{E}$ such that, $\left[e_{1}, e_{2}\right]=\left[e_{1}, T z\right]=[z, T z]=\mathbf{F}_{\mathbf{2}}$ and $e_{2} \perp_{t_{2}} \mathbf{F}_{\mathbf{1}}=\left[e_{1}\right] . T^{2} z \in \mathbf{E} \backslash \mathbf{F}_{\mathbf{2}}$, then by Lemma 11, again, there exists $e_{3} \in \mathbf{E}$ such that, $\left[z, T z, e_{3}\right]=\left[z, T z, T^{2} z\right]$ and $e_{3} \perp_{t_{3}}[z, T z]$. Hence, $\left[e_{3}, e_{2}, e_{1}\right]=\left[z, T z, T^{2} z\right]$ and $e_{3} \perp_{t_{3}}\left[e_{1}, e_{2}\right]=\mathbf{F}_{\mathbf{2}}$.

Continuing on this direction, we construct a sequence $\left(e_{n}\right)_{n}$ in $\mathbf{E}$ such that for each $n \geq 1$ we have:

$$
\begin{aligned}
\mathbf{F}_{\mathbf{n}}=\left[e_{1}, \ldots, e_{n}\right] & =\left[z, T z, \ldots, T^{n-1} z\right], \\
e_{n} \perp_{t_{n}}\left[e_{1}, \ldots, e_{n-1}\right] & =\mathbf{F}_{\mathbf{n}-\mathbf{1}} .
\end{aligned}
$$

Then, using Lemma 13 for each $n \geq 2$, we conclude that $\left(e_{m}\right)_{1 \leq m \leq n}$ is $\left(t_{2} \cdots t_{n}\right)$-orthogonal, so $\left(e_{n}\right)_{n \geq 1}$ is a $t$-orthogonal sequence in $\mathbf{E}$, and

$$
\overline{\left[e_{1}, e_{2}, \ldots\right]}=\overline{\left[z, T z, T^{2} z, \ldots\right]} .
$$

Hence, by Theorem 12, $\left(e_{n}\right)_{n}$ is a $t$-orthogonal basis of E. And for each $n \geq 1$ we have:

$$
\begin{aligned}
T\left(\mathbf{F}_{\mathbf{n}}\right) & =T\left[e_{1}, \ldots, e_{n}\right]=T\left[z, T z, \ldots, T^{n-1} z\right] \subseteq\left[z, T z, \ldots, T^{n} z\right] \\
& =\mathbf{F}_{\mathbf{n}+\mathbf{1}}=\left[e_{1}, \ldots, e_{n+1}\right] .
\end{aligned}
$$

Now, let show that $\liminf _{n} d\left(T\left(e_{n}\right),\left[e_{1}, \ldots, e_{n}\right]\right)=0$. Since for each sequence $\left(\lambda_{n}\right)_{n}$ of nonzero scalars, $\left(\lambda_{n} e_{n}\right)_{n}$ is a $t$-orthogonal basis of $\mathbf{E}$ such that for each $n \geq 1$

$$
T\left[\lambda_{1} e_{1}, \ldots, \lambda_{n} e_{n}\right] \subseteq\left[\lambda_{1} e_{1}, \ldots, \lambda_{n+1} e_{n+1}\right],
$$

without loss of generality we can assume, that the $t$-orthogonal basis $\left(e_{n}\right)_{n}$ satisfies $|\rho| \leq\left\|e_{n}\right\| \leq 1$ for all $n \geq 1$ for some scalar $\rho$ such that $0<|\rho|<1$.

Let $n \geq 1, e_{n} \in\left[z, T z, \ldots, T^{n-1} z\right]=\mathbf{F}_{\mathbf{n}-\mathbf{1}}+\left[T^{n-1} z\right]$. Then, there exist $x_{n} \in \mathbf{F}_{\mathbf{n}-\mathbf{1}}$ and $\lambda_{n} \in \mathbb{K} \backslash\{0\}$ such that

$$
e_{n}=x_{n}+\lambda_{n} T^{n-1} z \text {. }
$$

Hence, $T e_{n}=T x_{n}+\lambda_{n} T^{n} z$. So, we have:

$$
\begin{aligned}
d\left(e_{n}, \mathbf{F}_{\mathbf{n}-\mathbf{1}}\right) & =d\left(\lambda_{n} T^{n-1} z, \mathbf{F}_{\mathbf{n}-\mathbf{1}}\right), \\
d\left(T e_{n}, \mathbf{F}_{\mathbf{n}}\right) & =d\left(\lambda_{n} T^{n} z, \mathbf{F}_{\mathbf{n}}\right) .
\end{aligned}
$$


Therefore, we have

$$
\begin{aligned}
d\left(e_{n+1}, \mathbf{F}_{\mathbf{n}}\right) & =d\left(\lambda_{n+1} T^{n} z, \mathbf{F}_{\mathbf{n}}\right)=\left|\lambda_{n+1}\right| d\left(T^{n} z, \mathbf{F}_{\mathbf{n}}\right), \\
d\left(T e_{n}, \mathbf{F}_{\mathbf{n}}\right) & =\left|\lambda_{n}\right| d\left(T^{n} z, \mathbf{F}_{\mathbf{n}}\right) .
\end{aligned}
$$

Hence,

$$
\begin{aligned}
d\left(T e_{n}, \mathbf{F}_{\mathbf{n}}\right) & =\frac{\left|\lambda_{n}\right|}{\left|\lambda_{n+1}\right|} d\left(e_{n+1}, \mathbf{F}_{\mathbf{n}}\right) \\
& \geq \frac{\left|\lambda_{n}\right|}{\left|\lambda_{n+1}\right|} t|| e_{n+1} \| \geq t|\rho| \frac{\left|\lambda_{n}\right|}{\left|\lambda_{n+1}\right|}
\end{aligned}
$$

Then, by (6), we have $d\left(\lambda_{n} T^{n} z, \mathbf{F}_{\mathbf{n}}\right) \geq \frac{\left|\lambda_{n}\right|}{\left|\lambda_{n+1}\right|} t|\rho| \Rightarrow \frac{t|\rho|}{\left|\lambda_{n+1}\right|} \leq\left\|T^{n} z\right\|$. So, by (2), $\liminf _{n}\left(\frac{1}{\left|\lambda_{n+1}\right|}\right)^{\frac{1}{n}}=0$. Since for all $n \geq 1$

$$
\left(\prod_{k=1}^{n} \frac{\left|\lambda_{k}\right|}{\left|\lambda_{k+1}\right|}\right)^{\frac{1}{n}}=\left(\frac{\left|\lambda_{1}\right|}{\left|\lambda_{n+1}\right|}\right)^{\frac{1}{n}}
$$

we have

$$
\liminf _{n}\left(\prod_{k=1}^{n} \frac{\left|\lambda_{k}\right|}{\left|\lambda_{k+1}\right|}\right)^{\frac{1}{n}}=0 .
$$

Then, there exists a subsequence $\left(\frac{\left|\lambda_{k_{j}}\right|}{\left|\lambda_{k_{j}+1}\right|}\right)_{j}$ of $\left(\frac{\left|\lambda_{k}\right|}{\left|\lambda_{k+1}\right|}\right)_{k}$ such that,

$$
\lim _{j}\left(\frac{\left|\lambda_{k_{j}}\right|}{\left|\lambda_{k_{j}+1}\right|}\right)=0
$$

On the other hand, by (7), for each $j \geq 1$ we have

$$
\begin{aligned}
d\left(T e_{k_{j}}, \mathbf{F}_{\mathbf{k}_{\mathbf{j}}}\right) & =\frac{\left|\lambda_{k_{j}}\right|}{\left|\lambda_{k_{j}+1}\right|} d\left(e_{k_{j}+1}, \mathbf{F}_{\mathbf{k}_{\mathbf{j}}}\right) \\
& \leq \frac{\left|\lambda_{k_{j}}\right|}{\left|\lambda_{k_{j}+1}\right|}\left\|e_{k_{j}+1}\right\| \leq \frac{\left|\lambda_{k_{j}}\right|}{\left|\lambda_{k_{j}+1}\right|} .
\end{aligned}
$$

Then, $\lim _{j} d\left(T e_{k_{j}}, \mathbf{F}_{\mathbf{k}_{\mathbf{j}}}\right)=0$. And $\liminf _{n} d\left(T\left(e_{n}\right), \mathbf{F}_{\mathbf{n}}\right)=0$. 
In the classical analysis, an operator $T$ acting on complex Hilbert space $\mathbf{H}$ is said to be triangular, if there exists a sequence $\left\{P_{n}\right\}$ of (orthogonal) projections of finite rank on $\mathbf{H}$ converging strongly to $I$ and satisfying $\left(I-P_{n}\right) T P_{n}=0$. That is equivalent, that $T$ have an upper triangular matrix with respect to some orthonormal basis of $\mathbf{H}$ [11].

In the same context, in a non-Archimedean Banach space of countable type, we give the following definition:

Definition 15. Let $T$ be a bounded operator on $\mathbf{E}$. We say $T$ is a triangular operator, if there exist $t \in] 0,1]$ and a $t$-orthogonal basis $\left(e_{n}\right)_{n}$ of $\mathbf{E}$, such that for each $n \geq 1,\left[e_{1}, e_{2}, \ldots, e_{k_{n}}\right]$ is invariant for $T$, where $\left(k_{n}\right)_{n}$ is a strictly increasing sequence in $\mathbb{N}^{*}$.

Remark 16 . If $\mathbb{K}$ is algebraically closed, every triangular operator is an upper triangular operator (have an upper triangular matrix in such a $t$ orthogonal basis). Indeed, for all $n \geq 1$, let $\mathbf{F}_{\mathbf{n}}=\left[\mathbf{e}_{\mathbf{1}}, \mathbf{e}_{\mathbf{2}}, \ldots, \mathbf{e}_{\mathbf{k}_{\mathbf{n}}}\right]$ and $\mathbf{F}_{\mathbf{0}}=$ $\{0\}$. Since $T\left(\mathbf{F}_{\mathbf{n}}\right) \subseteq \mathbf{F}_{\mathbf{n}}$ for all $n \in \mathbb{N}$, then by the well known result, that every operator in finite dimensional space over $\mathbb{K}$ is upper triangular, we have for all $n$ the quotient operator $S_{n}$ defined in $\mathbf{F}_{\mathbf{n}+\mathbf{1}} / \mathbf{F}_{\mathbf{n}}$ by $S_{n}(\bar{x})=S(x)$ for all $\bar{x} \in \mathbf{F}_{\mathbf{n}+\mathbf{1}} / \mathbf{F}_{\mathbf{n}}$, is upper triangular. So obviously there is $\left(b_{n}\right)_{n} \subseteq \mathbf{E} \backslash\{\mathbf{0}\}$ such that $\left[b_{1}, b_{2}, \ldots\right]=\mathbf{E}$ with the property that $T\left(\left[b_{1}, \ldots, b_{n}\right]\right) \subseteq\left[b_{1}, \ldots, b_{n}\right]$ for all $n \in \mathbb{N}^{*}$. Therefore, according to the same first part of proof of Lemma 14. we can construct a $t$-orthogonal sequence $\left(l_{n}\right)_{n}$ where $\left.t \in\right] 0,1[$, such that $\left[b_{1}, \ldots, b_{n}\right]=\left[l_{1}, \ldots, l_{n}\right]$ for all $n \in \mathbb{N}^{*}$. So $T\left(\left[l_{1}, \ldots l_{n}\right]\right) \subseteq\left[l_{1}, \ldots, l_{n}\right]$ for all $n \in \mathbb{N}^{*}$ and $\left(l_{n}\right)_{n}$ is $t$-orthogonal basis of $\mathbf{E}$, hence we have $\mathrm{T}$ is upper triangular operator.

Now, we state our second main result.

TheOREM 17. Let $T$ be a bounded operator on $\mathbf{E}$. If there exists a cyclic $T$-quasi null vector in $\mathbf{E}$, then $T$ can be written as the sum of triangular operator and a compact shift operator, each one of them possesses a nontrivial invariant closed subspace.

Proof. Let $t \in] 0,1\left[\right.$. By Lemma 14 , there exists $\left(e_{n}\right)_{n}$, a $t$-orthogonal basis of $\mathbf{E}$ such that $T\left(\mathbf{F}_{\mathbf{n}}\right) \subseteq \mathbf{F}_{\mathbf{n}+\mathbf{1}}$ and $\liminf _{n} d\left(T e_{n}, \mathbf{F}_{n}\right)=0$, where $\mathbf{F}_{\mathbf{n}}:=$ $\left[e_{1}, \ldots, e_{n}\right]$ for all $n \geq 1$. Without loss of generality, we can assume that $|\rho| \leq\left\|e_{n}\right\| \leq 1$ for all $n \geq 1$ for some scalar $\rho$ with $0<|\rho|<1$. Then, there exists a sequence $\left(k_{j}\right)_{j}$ in $\mathbb{N}$ such that $\lim _{j} d\left(T e_{k_{j}}, \mathbf{F}_{k_{j}}\right)=0$. 
For each $n \geq 1, T e_{n} \in \mathbf{F}_{n}+\left[e_{n+1}\right]$. So, there exist $y_{n} \in \mathbf{F}_{\mathbf{n}}$ and $\lambda_{n} \in \mathbb{K}$ such that $T e_{n}=y_{n}+\lambda_{n} e_{n+1}$. For each $j \geq 1$,

$$
d\left(\lambda_{k_{j}} e_{k_{j}+1}, \mathbf{F}_{\mathbf{k}_{\mathbf{j}}}\right) \geq t\left\|\lambda_{k_{j}} e_{k_{j}+1}\right\| \geq t\left|\lambda_{k_{j}}\right||\rho| .
$$

Then, $\lim _{j}\left|\lambda_{k_{j}}\right|=0$.

Let $M_{T}$ be the matrix of $T$ in the basis $\left(e_{n}\right)_{n}$. For each $n \geq 1$, let $y_{n}=$ $\lambda_{1}^{n} e_{1}+\cdots+\lambda_{n}^{n} e_{n},\left(\lambda_{1}^{n}, \ldots, \lambda_{n}^{n}\right) \in \mathbb{K}^{n}$. Then $M_{T}$ is given by:

$$
M_{T}=\left(\begin{array}{ccccc}
\lambda_{1}^{1} & \lambda_{1}^{2} & \lambda_{1}^{3} & \ldots & \ldots \\
\lambda_{1} & \lambda_{2}^{2} & \lambda_{2}^{3} & \ldots & \ldots \\
0 & \lambda_{2} & \lambda_{3}^{3} & \ldots & \ldots \\
0 & 0 & \lambda_{3} & \ldots & \ldots \\
0 & 0 & 0 & \ldots & \ldots \\
\ldots & \ldots & \ldots & \ldots & \ldots \\
\ldots & \ldots & \ldots & \ldots & \ldots
\end{array}\right) .
$$

Let $S$ be the operator defined on $\mathbf{E}$ by:

$$
S e_{n}:=\left\{\begin{array}{ll}
T e_{n} & \text { if } n \neq k_{j}, \\
y_{n} & \text { if } n=k_{j} .
\end{array} \quad(\forall n \geq 1) .\right.
$$

It is clear that the matrix of $S$ in the basis $\left(e_{n}\right)_{n}$ is obtained from $M_{T}$ by putting 0 in the place of $\lambda_{k_{j}}$ for all $j \geq 1$.

Consider the operator $U=T-S$ which is defined by:

$$
U e_{n}:=\left\{\begin{array}{ll}
0 & \text { if } n \neq k_{j}, \\
\lambda_{k_{j}} e_{k_{j}+1} & \text { if } n=k_{j} .
\end{array} \quad(\forall n \geq 1) .\right.
$$

$U$ is a shift operator on $\mathbf{E}$, and for each $x=\sum_{n=1}^{+\infty} x_{n} e_{n}$,

$$
U(x)=\sum_{j=1}^{+\infty} \lambda_{k_{j}} x_{k_{j}} e_{k_{j}+1} .
$$

We obviously have that $\left[e_{1}, e_{2}, \ldots, e_{k_{j}}\right]$ is invariant under $S$ for all $j$. Then, $S$ is a triangular operator. It remains to show that $U$ is compact.

For each $j \geq 2$, let $U_{j}:=P_{j} U$, where $P_{j}$ is a projection on $\mathbf{F}_{k_{j}}$ parallel to $\overline{\left[e_{k_{j}+1}, e_{k_{j}+2}, \ldots\right]}$. It is clear that $U_{j}$ is a finite rank operator. Let $x=$ 
$\sum_{n=1}^{+\infty} x_{n} e_{n} \in \mathbf{E} \backslash\{0\}$. For each $\epsilon>0$, there exists $j_{0} \geq 1$ such that $\left|\lambda_{k_{j}}\right| \leq \frac{t}{\|x\|} \epsilon$ for all $j \geq j_{0}$. Then, for each $j \geq j_{0}$, we have

$$
\begin{aligned}
\left\|U(x)-U_{j}(x)\right\| & =\left\|\sum_{r \geq j}^{+\infty} \lambda_{k_{r}} x_{k_{r}} e_{k_{r}+1}\right\| \leq \sup _{r \geq j}\left|\lambda_{k_{r}}\left\|x_{k_{r}} \mid\right\| e_{k_{j}+1} \|\right. \\
& \leq \frac{t}{\|x\|} \epsilon \sup _{r \geq j}\left|x_{k_{r}}\right| \leq \frac{t}{\|x\|} \epsilon \frac{\|x\|}{t}=\epsilon .
\end{aligned}
$$

Hence, $U=\lim _{j} U_{j}$. Therefore, $U$ is a compact operator.

As a consequence of Theorem [17, we conclude the following important result.

COROLlary 18. If $\mathbb{K}$ is algebraically closed, then every compact operator in $\mathbf{E}$ either has a nontrivial invariant closed subspace or is a sum of two compact operators, one is upper triangular and the second one is a shift.

Proof. Let $T$ be a compact operator in $\mathbf{E}$, we assume that the set of points of the spectrum of $T$ is equal to $\{0\}$ and $T$ has a cyclic vector (otherwise $T$ has a nontrivial closed invariant subspace (see [17, p. 15]), then with [17, Theorem 6.14], we have $\lim _{n}\left\|T^{n}\right\|^{\frac{1}{n}}=0$, so $\lim _{n}\left\|T^{n} z\right\|^{\frac{1}{n}}=0$ for all $z \in \mathbf{E}$. This, with Remark 16 and Theorem 17 completes the proof.

\section{ACKNOWLEDGEMENTS}

The authors especially thank the referees for their valuable suggestions in preparing this paper.

\section{REFERENCES}

[1] Y.A. Abramovich, C.D. Aliprantis, O. Burkinshaw, Invariant subspaces of operators on $l_{p}$-spaces, J. Funct. Anal. 115 (2) (1993), 418-424.

[2] Y. Amice, Interpolation p-adique, Bull. Soc. Math. France 92 (1964), 117 180.

[3] N. Aronszajn, K.T. Smith, Invariant subspaces of completely continuous operators, Ann. of Math. (2) (60) (1954), 345-350.

[4] W.B. Arveson, J. Feldman, A note on invariant subspace, Michigan Math. J. 15 (1968), 61-64.

[5] A.R. Bernstein, A. Robinson, Solution of an invariant subspace problem of K.T. Smith and P.R. Halmos, Pacific J. Math. 16 (1966), 421-431. 
[6] A. Beurling, On two problems concerning linear transformations in Hilbert space, Acta Math. 81 (1948), 239-255.

[7] R.L. ElLis, The Fredholm alternative for non-Archimedean fields, J. London Math. Soc. 42 (1967), 701-705.

[8] L. Gruson, Théorie de Fredholm p-adique, Bull. Soc. Math. France 94 (1966), $67-95$.

[9] D.W. Hadwin, E.A. Nordgren, H. Radjavi, P. Rosenthal, An operator not satisfying Lomonosov's hypothesis, J. Functional Analysis 38 (1980), $410-115$.

[10] P.R. Halmos, Invariant subspaces of polynomially compact operators, Pacific J. Math. 16 (1966), 433-437.

[11] P.R. Halmos, Quasitriangular operators, Acta Sci. Math. (Szeged) 29 (1968), $283-293$.

[12] V. Lomonosov, Invariant subspaces of the family of operators that commute with a completely continuous operator (Russian), Funkcional. Anal. i Priložen 7 (1973), 55-56.

[13] A.J. MichaEL, Hilden's simple proof of Lomonosov's invariant subspace theorem, Adv. Math. 25 (1977), 56-58.

[14] C. Pearcy, N. Salinas, An invariant-subspace theorem, Michigan Math. J. 20 (1973), $21-31$.

[15] C. Perez-Garcia, W.H. Schikhof, "Locally Convex Spaces over NonArchimedean Valued Fields", Cambridge Studies in Advanced Mathematics 119, Cambridge University Press, Cambridge, 2010.

[16] H. Radjavi, P. Rosenthal, "Invariant Subspaces", Springer-Verlag, New York-Heidelberg, 1973.

[17] W.H. Schikнof, On p-adic compact operators, Report 8911, Department of mathematics, Catholic University, Nijmegen, The Netherlands, 1989, 1-28.

[18] P. SCHNEIDER, "Nonarchimedean Functional Analysis", Springer-Verlag, Berlin, 2002.

[19] J.P. SERRE, Endomorphismes complètement continus des espaces de Banach p-adiques, Inst. Hautes Études Sci. Publ. Math. 12 (1962), 69-85.

[20] W. ŚliwA, The invariant subspace problem for non-Archimedean Banach spaces, Canad. Math. Bull. 51 (2008), 604-617.

[21] A.C.M. van RoolJ, "Non-Archimedean Functional Analysis", Monographs and Textbooks in Pure and Applied Math. 51, Marcel Dekker, Inc., New York, 1978. 